Service social

\title{
Des services de soutien à domicile fragiles pour une clientèle vulnérable
}

\section{Mireille Dumont-Lemasson}

Volume 43, numéro 1, 1994

Maintien à domicile

URI : https://id.erudit.org/iderudit/706642ar

DOI : https://doi.org/10.7202/706642ar

Aller au sommaire du numéro

\section{Éditeur(s)}

École de service social de l'Université Laval

ISSN

1708-1734 (numérique)

Découvrir la revue

Citer cet article

Dumont-Lemasson, M. (1994). Des services de soutien à domicile fragiles pour une clientèle vulnérable. Service social, 43(1), 47-65.

https://doi.org/10.7202/706642ar
Résumé de l'article

Les services à domicile offerts par les CLSC constituent le maillon stratégique des services socio-sanitaires de soutien à domicile. Cet article met en évidence le grave sous-financement de ces services et montre quelles stratégies d'adaptation sont déjà à l'œuvre. L'approche communautaire et un réalignement de l'ensemble des services socio-sanitaires apparaissent à cet égard les seules voies capables de réconcilier les nouvelles contraintes et les valeurs de base. Dans la mesure où, à l'avenir, la nécessité d'améliorer les services offerts va s'imposer, de même que celle de répondre de manière plus diversifiée aux besoins, l'alliance avec le milieu communautaire apparaît incontournable. La promotion des conditions politiques favorisant l'émergence d'une politique intersectorielle de soutien à domicile devrait mobiliser les CLSC. 


\section{Des services de soutien à domicile fragiles pour une clientèle vulnérable}

Mireille DUMONT-LEMASSON

Conseillère-cadre

Fédération des CLSC du Québec

Au Québec, parmi l'ensemble des secteurs touchés par le vieillissement de la population et par la nécessité de promouvoir un ensemble de mesures facilitant un meilleur "vieillir chez soi», il revient au ministère de la Santé et des Services sociaux (MSSS) et plus récemment à la Société d'habitation du Québec d'avoir fait œuvre de pionniers dans ce domaine et posé les premiers jalons d'une politique de soutien à domicile. Mais il s'agit d'un début fort timide, alors que la société québécoise devra, dans les vingt prochaines années, adapter ses politiques sociales - autant sur le plan du revenu, du logement, du transport que de l'aménagement urbain - à sa population aînée plus nombreuse et dont l'espérance de vie s'accroît. Le rapport récent du groupe d'experts sur le vieillissement, Vers un nouvel équilibre des âges, incite l'ensemble des acteurs à définir des actions concertées et intersectorielles. Il lève le voile sur l'hétérogénéité des conditions de vie des aînés sur les plans économique, sanitaire et social et, en conséquence, sur la nécessaire réévaluation de l'ensemble des dispositifs sociaux 
incluant les services socio-sanitaires. Mais le temps ne semble pas encore mûr pour l'établissement d'une politique gouvernementale de soutien à domicile, puisque la seule retombée tangible de ce rapport a été, en 1993, la création du Conseil des aînés par lequel, sur un mode consultatif, les aînés pourront faire entendre leurs voix sur les mesures gouvernementales qui les concernent.

Depuis une dizaine d'années, le ministère de la Santé et des Services sociaux a défini des orientations qui affirment clairement I'importance de soutenir les aînés dans leur milieu de vie naturel' ${ }^{1}$. Pour les personnes en perte d'autonomie, différents services ont été instaurés, particulièrement des services à domicile, des centres de jour et, dans le domaine hospitalier, des unités d'expertise gériatrique. Environ 350 organismes communautaires offrant des services bénévoles ont reçu des appuis financiers pour assurer leur infrastructure de base. Les récentes orientations du MSSS ${ }^{2}$ préconisent un rehaussement des budgets de 200 millions de dollars en cinq ans pour accroître les services offerts directement dans le milieu de vie. II s'agit notamment de rendre plus accessibles les services à domicile à l'ensemble des aînés, y compris ceux qui vivent en résidences privées et en habitations à loyer modique, les mesures de répit et de dépannage pour soulager les familles aidantes, de même que la fréquentation des centres de jour, et de consolider ou susciter l'émergence de ressources bénévoles. Enfin, un des dix-neuf objectifs de la politique de santé et de bien-être ${ }^{3}$ est consacré à la réduction des obstacles à l'intégration sociale des personnes âgées dans la société.

Dans ce panorama de ressources, les services à domicile offerts par les CLSC du Québec forment le maillon stratégique des services publics consacrés au soutien à domicile des aînés. Il s'agit d'une combinaison de services diversifiés, tels que l'aide et l'assistance physique dans I'entretien du domicile, la préparation des repas et les soins personnels, les soins infirmiers et médicaux, la réadaptation, I'assistance psychosociale dans des situations personnelles et sociales difficiles autant pour la personne vieillissante que pour ses proches, la médiation et la liaison avec les ressources environnantes aussi bien institutionnelles que bénévoles ou encore le soutien au développement de projets locaux à caractère communautaire.

1. Nous renvoyons ici aux politiques officielles Pour mieux vieillir au Québec (1980) et Un nouvel âge à partager (1985), émanant du ministère des Affaires sociales.

2. Gouvernement du Québec, MSSS, Une réforme axée sur le citoyen (1990), p. 26 et 27.

3. Gouvernement du Québec, MSSS, La politique de la santé et du bien-être (1992), p. 118-122. 
Les CLSC rejoignent un nombre important de personnes âgées qui forment la clientèle majoritaire mais non exclusive des services offerts à domicile. Au cours de I'année 1990-1991, selon les derniers chiffres officiels disponibles ${ }^{4}$, au moins 79500 personnes de plus de 65 ans ont reçu des services à domicile, dont près de 8000 de manière intensive. Globalement, ces services rejoignent $10,5 \%$ de la population âgée de 65 ans et plus, dont 16,7\% de celle qui a franchi le cap des 75 ans.

L'objectif premier de ces services est de soutenir I'autonomie de la personne, son intégrité sociale liée au maintien dans son environnement familier et de minimiser ou d'éviter les effets néfastes d'une hospitalisation. C'est aussi d'éviter ou de retarder l'éventualité d'un hébergement de longue durée qui, malgré les meilleurs efforts, réduit inévitablement les capacités d'agir des personnes et leur insertion sociale.

Ces services s'adressent donc à ceux et celles dont l'état de vulnérabilité grandit dans les gestes quotidiens et les contacts sociaux. En corollaire, pour la majorité d'entre eux, s'accroissent l'insécurité devant le vieillissement, les sentiments de solitude et d'inutilité. Bien que le quart de la clientèle rejointe vive seule, la majorité reçoit, à des degrés divers, l'aide de son conjoint ou de sa conjointe ou de membres de sa famille immédiate. Dans ce cas, les services visent à appuyer l'effort des proches et à prendre la relève dans certaines tâches difficiles.

Financés à même les fonds publics, ces services devraient constituer, à notre avis, une des pierres angulaires d'une politique de vieillissement qui prend en compte les besoins sociaux des personnes de grand âge dépendantes de l'aide d'autrui. C'est dans cette perspective, à partir des travaux réalisés par le MSSS et dans le réseau, que nous ferons l'analyse de la situation actuelle et que nous situerons les enjeux futurs. Même si ce point de vue peut paraître sombre, nous souhaitons que les mesures de soutien pour ces personnes dans la communauté deviennent un enjeu social. À divers titres, chacune et chacun, nous avons à témoigner qu'il est humainement souhaitable, pertinent, réalisable, voire rentable que les aînés aient accès à un ensemble de services diversifiés et de qualité, qui assurent leur sécurité et leur bienêtre dans leur milieu familier.

4. L. Marcotte, Analyse de la cueillette d'information en maintien à domicile réalisée auprès des CLSC pour l'année financière 1990-1991, MSSS, Direction des programmes à la communauté, mai 1993. Note complémentaire: le taux de réponse est de $70 \%$ des CLSC du Québec. 


\section{SITUATION ACTUELLE DES SERVICES À dOMICILE}

\section{Des orientations à concrétiser}

Depuis la mise en œuvre des services à domicile, un premier document officiel a été produit par le ministère de la Santé et des Services sociaux (MSSS) pour situer les objectifs et les conditions d'accès aux services à domicile publics. Il s'agit de la Politique de services à domicile, publiée en 1979; celle-ci, dans une optique essentiellement préventive, garantissait leur universalité et l'accès aux soins infirmiers et à I'aide familiale. En 1992, le MSSS a élaboré un projet de révision de la politique, qui a fait l'objet d'une large consultation. La version définitive a été rendue publique en mai 1994 sous le titre Les services à domicile de première ligne: cadre de référence. Enfin, un plan d'action du MSSS pour les personnes âgées, qui faisait suite aux recommandations du groupe d'experts sur le vieillissement (1991), est également en chantier.

Alors que les besoins de services ont évolué en diversité et en ampleur, les orientations proposées restent, aux yeux des clientèles et des artisans, généreuses mais peu précises. Quels sont les objectifs à poursuivre? Soutenir l'intégration sociale ou prévenir le recours aux services les plus coûteux, dont l'hébergement et les soins hospitaliers? Quel objectif doit avoir la priorité? Quelles en sont les conditions de mise en œuvre, notamment le financement? Quelles en sont les limites? Doit-on répondre aux personnes qui ont les besoins les plus grands ou soutenir, par des actions ponctuelles, les personnes en moins grande perte d'autonomie, mais qui, avec un coup de pouce, resteraient plus en sécurité et fonctionnelles dans leur milieu? En fonction de quels critères les services, les équipements et les fournitures seront-ils couverts d'une façon universelle, même si ce principe est désormais reconnu? Le soutien aux tâches domestiques étant souvent l'objet de demandes de services, car il est un révélateur d'un début de difficulté pour la personne âgée, doit-il être assuré par le CLSC? par d'autres organismes? universellement ou conditionnellement au revenu des personnes? Quels sont les paramètres qui guident I'octroi $d^{\prime}$ une aide financière à l'usager ou à sa famille, lui permettant de pourvoir lui-même à l'engagement de personnel pour certains services plutôt que de les recevoir par le personnel du CLSC?

Ces questions fondamentales, encore sans réponse, touchent à la finalité, à l'équité, à l'universalité, à l'efficience et au caractère public des services à domicile. Les seules hypothèses plausibles qui peuvent expliquer cet attentisme ministériel nous paraissent relever d'une méconnaissance relative de la part des décideurs, des besoins d'une 
clientèle silencieuse qui n'a pas de mécanisme politique pour témoigner de sa condition; de la faible reconnaissance des services sociaux et notamment des services non professionnels dans le panier des services assurés par l'État; d'un manque flagrant de planification intégrée des programmes destinés aux aînés au sein même du Ministère et des régies régionales; et enfin de la difficulté de faire des choix de réallocation de ressources du milieu institutionnel vers la communauté.

\section{Le sous-financement chronique}

Si l'on observe I'effort financier du MSSS en matière de services à domicile, ce secteur d'activité socio-sanitaire semble avoir connu une croissance significative au cours des dix dernières années. Globalement, les crédits alloués aux services à domicile et organismes communautaires seulement ont crû, passant de 44,4 millions de dollars en 1979-1980 à 329 millions en 1993-19945. Cette augmentation reflète principalement le transfert de ressources déjà existantes en centres hospitaliers et en centres de services sociaux vers les CLSC et l'indexation annuelle des budgets. L'injection de sommes nouvelles dans les services à domicile s'est produite après 1986, avec l'adoption du plan de désengorgement des salles d'urgence hospitalières et l'instauration d'un budget de développement pour faire face à l' " alourdissement » de la clientèle âgée.

La part relative des investissements publics dans I'ensemble des services liés au soutien à domicile est cependant demeurée marginale. Selon le groupe d'experts sur le vieillissement, l'ensemble des programmes dits de maintien à domicile (services à domicile, centres de jour et organismes communautaires) représentait, en 1989-1990, 8,4\% des budgets consacrés aux personnes âgées dans le secteur de l'hébergement intermédiaire et de longue durée et de l'expertise gériatrique ${ }^{6}$. Ce pourcentage se réduit à environ $2 \%$ de l'ensemble des dépenses du MSSS consacrées aux aînés si l'on inclut l'ensemble des soins hospitaliers et médicaux et les médicaments.

5. Gouvernement du Québec, MSSS, Les services à domicile de première ligne: cadre de référence, mai 1994, p. 7. Ce budget comprend 192,6 millions de dollars pour les services réguliers et intensifs dans les CLSC, 12,9 millions pour les organismes communautaires, 25,5 millions pour les fonds régionaux pour les personnes handicapées et 7,9 millions pour les services de soutien aux familles des personnes handicapées.

6. Gouvernement du Québec, MSSS, Vers un nouvel équilibre des âges. Rapport du groupe d'experts sur le vieillissement, 1991, p. 59. 
Entre les années 1988-1989 et 1992-1993, le Ministère a investi globalement 142,1 millions de dollars pour faire face à l'alourdissement de la clientèle âgée vivant en centres d'hébergement publics et à domicile. Cet ajout a été globalement inférieur dans le secteur du maintien à domicile comparativement à celui de I'hébergement. Ces secteurs ont reçu respectivement 68,2 millions de dollars et 73,9 millions pour les cinq années ${ }^{7}$.

En conséquence, les CLSC investissent plus qu'ils ne reçoivent dans le cadre du budget protégé de soutien à domicile, au détriment de leurs autres services, soit une ponction de 12 millions de dollars de leur budget global en 1991-19928, et ils doivent gérer les services dans un contexte de pénurie chronique.

De façon générale, le Québec reste une des provinces qui n'ont pas amorcé vraiment un virage favorable au soutien à domicile. Le per capita, pour l'ensemble des ressources de maintien à domicile (organismes communautaires, services à domicile en CLSC, soutien à la famille) en 1993-1994 représente 33,65 \$9. Comparativement, le per capita moyen des provinces canadiennes qui ont fait du programme de maintien à domicile un élément central des services de soutien aux aînés et aux personnes handicapées se situe autour de $60 \$$ et plus. Quelle que soit la méthode utilisée, tous conviennent que le financement actuel des services à domicile devrait au moins tripler pour atteindre un niveau acceptable.

L'expérience canadienne montre que, pour être efficient, un investissement plus important dans les services de soutien à domicile doit être jumelé à des restrictions d'accès aux ressources plus onéreuses, par l'instauration d'un point d'accès unique des demandes de services, et à la réallocation de ressources en provenance du secteur hospitalier et de l'hébergement vers les services à domicile. C'est le sens des réformes introduites au Manitoba, en Colombie-Britannique et plus récemment en Ontario $^{10}$.

7. FCLSCQ, Avis sur les services à domicile, présenté au ministre de la Santé et des Services sociaux le 24 mars 1993.

8. FCLSCQ, ibid.

9. MSSS, Cadre de référence, op. cit., p. 7. Le per capita de I'Ontario atteignait 66,48 \$ en 1992-1993, celui de la Suède 104,70 \$ en 1991.

10. Province de la Colombie-Britannique, Closer to Home, vol. 2, Rapport de la Commission royale d'enquête sur les coûts du système de santé, 1991 et Directions for a Healthy British Columbia, Ministry of Health, 1992 et Province de I'Ontario, Nouvelle orientation en matière de soins de longue durée et de services de soutien à domicile, ministère de la Santé de I'Ontario, octobre 1991. 
L'ampleur possible de la substitution à moindre coût est toujours source d'opinions variées. En effet, les études disponibles ne prennent pas toujours en compte les mêmes profils de clientèle, des services comparables ou les variables reliées à l'organisation des services. Toutefois, à certaines conditions, les services à domicile pourraient avoir trois grands effets sur la réduction des coûts majeurs dans le système socio-sanitaire :

a) La réduction du nombre de personnes recourant pour des raisons sociales et de sécurité à l'hébergement ${ }^{11}$ (au moins 16,4\% des personnes au Québec sont hébergées alors que leur profil de besoins ne le justifierait pas); il y aurait là une économie significative dans la mesure où il en coûte autour de $30000 \$$ par an pour être hébergé. Actuellement, le coût d'une place en services à domicile intensifs est de l'ordre de 10000 \$.

b) La réduction de la durée et du taux d'hospitalisation par la mise en place de services palliatifs et de relève à domicile adéquats ; actuellement, le taux d'hospitalisation des personnes âgées est en hausse et trois personnes âgées sur cinq décèdent à l'hôpital $\left.\right|^{12}$.

c) La réduction du recours à l'urgence hospitalière par la mise sur pied d'un service téléphonique accessible en tout temps, qui assure I'orientation et les conseils infirmiers et sociaux aux personnes et à leurs familles dans le cas de crise aiguë. C'est ce que démontrent les études réalisées sur les services Info-santé accessibles 24 heures sur 24 tous les jours de la semaine dans les régions des Laurentides et de l'Outaouais. Sans ce service, les personnes se seraient présentées à l'urgence.

Le maintien de la contribution familiale dans l'aide aux aînés et le fait qu'un grand nombre de services nécessaires soient d'ordre non professionnel sont de toute évidence des dimensions essentielles à l'efficience tant recherchée des services à domicile.

\section{L'intensification des services}

Un consensus assez large existe au Québec sur le fait que l'adaptation des services à domicile aux besoins des personnes âgées ou des

11. Louise Garant, Synthèse d'un programme d'évaluation sur la réponse aux besoins de longue durée des personnes âgées ayant des limitations fonctionnelles, MSSS, Direction de l'évaluation, février 1994, p. 14.

12. M. Levasseur, La consommation de soins dans les centres hospitaliers de courte durée par les personnes âgées: Québec, 1981-1982, MSSS, Direction de la planification et de l'évaluation, 1992. 
adultes handicapés atteints d'incapacités sévères se conjugue nécessairement avec la possibilité d'offrir quotidiennement une plus grande assistance à la personne et à sa famille et une intervention professionnelle précoce pour prévenir autant la détérioration de la santé de la personne âgée que l'épuisement des proches. L'intensification des services est perçue comme un moyen facilitant la normalisation et I'intégration sociale des personnes et un frein à l'hospitalisation et à I'hébergement.

Après six ans de mise en œuvre de deux programmes de financement permettant l'intensification des services, I'un s'adressant aux personnes lourdement handicapées (Fonds régionaux pour personnes handicapées) et l'autre visant la clientèle âgée en attente d'hébergement ou en phase de post-hospitalisation et en phase palliative (services intensifs de maintien à domicile-SIMAD), nous sommes en mesure d'établir certains constats :

a) une plus grande acceptation du soutien à domicile de la part des personnes en perte d'autonomie sévère et de leurs proches ainsi que de la part des professionnels en CLSC et dans le réseau de la santé. Comme le souligne Joubert ${ }^{13}$ dans l'évaluation des services intensifs (SIMAD), "le recours à I'hébergement devient alors une alternative lorsque le maintien à domicile n'est plus désirable ou n'est plus souhaité" .

b) la réduction, grâce aux services à domicile, de l'utilisation des ressources institutionnelles, car ils permettent d'éviter les hospitalisations inappropriées et de retarder l'échéance d'un hébergement;

c) l'émergence de nouvelles formes d'organisation du répit pour les proches des personnes âgées et un certain allégement du fardeau des familles;

d) le décloisonnement des ressources, notamment celles qui œuvrent dans une finalité de maintien à domicile, particulièrement entre CLSC, organismes communautaires et centres de jour ;

e) I'accroissement du contrôle administratif par la régie régionale sur le programme local des CLSC en dépit d'un processus partiel de décentralisation des différentes enveloppes allouées pour les services intensifs aux personnes âgées, aux personnes en phase terminale, aux personnes lourdement handicapées à long terme et au répit-gardiennage; des différences régionales d'orientation

13. P. Joubert, A. Laberge, J.-P. Fortin, M. Paradis et F. Desbiens, Évaluation du programme québécois de services intensifs de maintien à domicile (SIMAD), Unité de recherche en santé communautaire, Centre hospitalier de l'Université Laval, 1991, p. 127. 
se répercutent notamment sur les critères $d^{\prime}$ accès aux services et sur les modes de prestation des services d'aide;

f) le développement d'une alternative privée à but lucratif et communautaire et de la pratique de subventionner l'usager pour fournir des services d'aide et d'assistance à moindre coût que s'ils étaient offerts par le personnel du CLSC. Dans les deux programmes de services intensifs existants, le taux horaire autorisé au niveau régional (et provincial) pour les services essentiels d'aide, $\mathrm{d}^{\prime}$ assistance et de gardiennage exclut de facto le mode de prestation des services par le personnel du CLSC, à moins que celui-ci opte clairement pour combler, à même ses propres fonds, le manque à gagner ;

g) la responsabilisation accrue des personnes les plus vulnérables dans la recherche de main-d'œuvre privée de services d'aide et $d^{\prime}$ assistance sans disposer de conditions permettant de recruter des personnes qualifiées et convenablement rémunérées;

h) le maintien d'un traitement différent pour la clientèle âgée et la clientèle adulte handicapée, même si les deux présentent des besoins comparables. Certains principes revendiqués par les personnes handicapées sont généralement préservés, notamment le respect du choix de vivre en dehors de l'établissement. De façon générale, la quantité de services n'est pas conditionnelle au revenu des personnes; le respect du plan de services en cas de déménagement et la protection du budget de services par client sont implicitement reconnus; advenant un manque budgétaire, ce qui est le cas actuellement, la création de listes d'attente est faite au niveau régional, permettant une possibilité de défense des besoins des personnes handicapées auprès du MSSS; les personnes représentant les usagers et I'OPHQ ont exercé un certain droit de regard sur les choix régionaux. Il existe d'ailleurs, dans presque toutes les régions, des regroupements régionaux associatifs qui exercent une vigilance importante sur tous ces aspects.

Dans le cas des personnes très âgées et fréquemment seules qui forment la clientèle presque exclusive des services intensifs (SIMAD), ces principes essentiels n'ont pas été clairement énoncés, ni mis en discussion.

Bref, I'intensification des services a créé un repositionnement complet des services à domicile. De façon très positive, ces services ont proposé de nouveaux choix aux aînés et à leurs familles et apporté une solution de rechange crédible à l'institutionnalisation. Mais en même temps les problèmes récurrents quant à l'accès, à la qualité et 
à l'équité des services remettent en question le pouvoir réel des aînés et de leurs représentants d'influencer les intervenants et les planificateurs pour améliorer la situation présente.

\section{Les stratégies d'adaptation}

Sur le plan local, la pénurie financière du programme entraîne la nécessité de faire des choix d'intervention et de gestion et d'établir en conséquence des priorités. Ces dernières peuvent porter sur les critères d'entrée au programme, sur les choix de services, sur les modes de prestation, sur le coût des services ou la recherche de solutions coopératives avec le milieu. Tous les intervenants et les gestionnaires élaborent forcément des stratégies d'adaptation dans un contexte de rareté. Nous exposerons les plus fréquentes. Chacune a ses forces et ses limites, certes, mais si elles se contredisent d'un CLSC à l'autre, à long terme cela risque de nuire à la crédibilité et à l'efficience des services.

\section{L'admissibilité aux services - la solution communautaire}

Toute demande de services est soumise à l'appréciation d'un professionnel qui procède à une évaluation pour déterminer l'admissibilité au service. Pour la prestation de soins infirmiers, I'ordonnance médicale justifie un accès universel aux soins; dans les autres services professionnels et les services d'aide, la réponse dépendra autant de la nature des besoins que de la disponibilité et de l'organisation des services.

De façon générale, les critères d'accès sont devenus très restrictifs, quel que soit le niveau de services offert par la suite. II existe souvent une certaine incohérence dans le fait de "privilégier» les personnes les plus vulnérables, alors que par ailleurs les moyens sont déficients pour assurer la qualité de vie de ces personnes, notamment par un encadrement plus soutenu sur le plan des tâches domestiques, de la santé, du transport, des repas, de la socialisation ou de la sécurité.

De nombreux CLSC ont, de ce fait, clairement opté pour soutenir l'émergence de ressources communautaires, notamment en faisant participer les aînés à une démarche de sensibilisation aux conditions favorables à un meilleur vieillissement. Les initiatives à ce chapitre témoignent de solutions prometteuses, comme: la participation des facteurs aux actions de vigilance, la mise sur pied de coopératives de services pour des services ménagers, la mise en œuvre de projets alimentaires, l'organisation de maisons de répit pour les proches, etc.

Enfin, une étroite collaboration locale se dessine dans certains milieux entre les établissements publics, privés et communautaires pour maximiser le potentiel de réponse aux besoins des aînés. 


\section{Le saupoudrage ou le guichet fermé}

Cette tendance à donner moins de services à plus de monde est, à notre avis, la plus fréquente. Elle évite de créer des listes d'attente qui, aux yeux de la population et des autres partenaires du réseau, peuvent signifier une absence d'efficience. Par contre, elle fragilise les services à un double point de vue: le plan de services peut être révisé en tout temps, la durée des services peut être écourtée, alors que la clientèle admise est, comme on l'a dit précédemment, en difficulté sérieuse.

Certains CLSC préfèrent créer des listes d'attente, même si elles créent de l'insatisfaction, pour garantir véritablement un service adéquat et conforme aux besoins des personnes. Ce faisant, ils doivent assumer les plaintes des usagers, celles des milieux hospitaliers qui leur envoient des personnes et trouver des solutions palliatives pour les personnes en situation de crise ou de détérioration subite de leur condition de santé.

\section{La privatisation}

Une autre stratégie d'adaptation consiste en la privatisation progressive des services d'aide, d'assistance et de gardiennage, soit par le truchement de l'allocation directe à l'usager ou par l'achat de services par le CLSC à des agences privées afin d'augmenter le volume de services à coût moindre.

À l'exception de la région de Montréal et de Laval, où la régie régionale avait déterminé des règles particulières privilégiant le recours aux agences et déterminé un taux horaire supérieur à celui déterminé par le Conseil du trésor, le taux horaire consenti pour l'allocation directe dans les autres régions se situe autour de 6,00 \$ I'heure et de $2,00 \$$ à 4,00\$ pour le répit-gardiennage. L'application de ces taux varie selon les balises régionales.

La pratique des subventions à l'usager s'est généralisée. On observe une plus grande utilisation de cette modalité même dans le cas des services d'aide courants traditionnellement offerts par le CLSC. Cette pratique touche $27 \%$ des services réguliers d'aide, $17 \%$ des services intensifs à la clientèle âgée. Le volume de services est quatre fois plus élevé que si le CLSC offrait lui-même ces services. En termes financiers, l'ensemble des fond alloués de cette façon représente de façon conservatrice une estimation de près de 30 millions de dollars en 1990$1991^{14}$ (pour l'ensemble de la clientèle) en incluant le soutien à la famille.

14. Mario Bolduc et Jocelyne Bélanger, Quelques données de base sur la formule de l'allocation directe, MSSS, Direction de l'évaluation. Document de travail, septembre 1993. 
Si dans les faits cette modalité permet d'assurer une plus grande quantité de services, elle soulève d'énormes questions d'éthique chez les gestionnaires et les intervenants. Parmi les plus importantes, notons

a) I'absence de véritable choix par l'usager d'être desservi par le CLSC ou de recevoir cette allocation;

b) la difficulté réelle de gérer pour les aînés et leurs proches, dans le respect des lois sociales, un lien d'emploi. En effet, le taux horaire consenti rend à toutes fins pratiques le recrutement de personnel qualifié difficile et pose de surcroît la question de la précarité des conditions de travail de ces employés non déclarés.

Il semble évident qu'une telle situation appelle des correctifs majeurs, car ce n'est plus une pratique d'exception justifiée par des circonstances particulières de dépannage, de souplesse des horaires, de disponibilité de personnel, mais par des considérations essentiellement économiques. On se trouve en présence de deux catégories de services d'aide. La première permet l'engagement de personnes de son choix, incluant parfois des membres de la parenté, pour effectuer des tâches simples et fréquentes, avec des horaires souples, mais dans des conditions quasi illégales. La seconde garantit la qualité et la supervision du personnel, l'adhésion à des objectifs multidisciplinaires; mais I'intensité du service est plus faible, il y a moins de souplesse dans I'horaire; les tâches sont plus centrées sur les soins personnels et l'intervention éducative dans des contextes familiaux plus conflictuels.

En somme, en I'absence d'une politique aux objectifs clairs et subissant un sous-financement chronique grave, les CLSC, sous la pression d'une demande croissante de services, s'adaptent comme ils le peuvent à une situation de plus en plus intenable. Alors que les besoins s'accroissent, ils sont conduits à créer iniquité et frustration, déqualification et mise en place de conditions de privatisation qui les menacent ou, dans les cas plus positifs, à faire appel à de nouvelles stratégies communautaires pour rendre plus acceptable une sélectivité croissante. II devient, dans cette perspective, capital de trouver des solutions satisfaisantes pour les années à venir.

\section{LES SOLUTIONS POSSIBLES}

\section{Miser sur la réforme de la santé et des services sociaux}

Plusieurs éléments de la Réforme, soit les orientations dévoilées en décembre 1990, I'adoption de la Loi sur l'organisation des services de santé et des services sociaux (1991) et la Politique pour la santé et le bien-être (1992), nous permettent de croire que certains facteurs 
devraient, à moyen terme, modifier des aspects majeurs concernant la lecture des besoins, I'allocation des ressources publiques, I'organisation des services publics et communautaires et les modes d'évaluation des services.

L'allocation des ressources financières sera conditionnelle à I'atteinte des objectifs de résultats en ce qui regarde l'amélioration de la santé et du bien-être, mais aussi l'efficience des producteurs de services. Enfin, les personnes âgées en perte d'autonomie feront l'objet d'un programme-clientèle qui, sur le plan régional, visera l'intégration des services autour de cette clientèle et, sur le plan provincial, I'intégration des perspectives et des modes de budgétisation en fonction de paramètres identiques. L'allocation de ressources devra permettre, au moins théoriquement, de diriger les efforts vers les lieux de services où les besoins sont les plus grands.

Les régies régionales disposeront de pouvoirs leur permettant de définir l'organisation des services la plus judicieuse et la plus efficiente possible pour répondre aux besoins et d'ajuster l'allocation de ressources en conséquence, même par transferts de ressources entre différents types d'établissements. Les décisions régionales seront davantage influencées par la population compte tenu de la composition du conseil d'administration, formé en parts égales d'élus municipaux, de personnes représentatives de groupes socio-économiques et des autres secteurs d'activité, des usagers membres de conseils d'administration de chaque catégorie d'établissements publics et privés, d'élus municipaux et de représentants provenant de divers types d'organismes communautaires. Cette nouvelle dynamique devrait permettre d'établir de nouvelles assises à la concertation intersectorielle ainsi qu'entre le secteur public et le secteur communautaire.

Par ailleurs, I'influence des usagers s'accroîtra vraisemblablement, dans la mesure où le traitement des plaintes doit faire l'objet dans chaque établissement d'une procédure structurée avec un mécanisme $\mathrm{d}$ 'appel pour les clients auprès du Commissaire aux plaintes au niveau de la Régie régionale.

De plus, I'obligation pour les établissements de définir, en collaboration avec le client et ses proches, des plans d'intervention et des plans de services pour les clientèles susceptibles de les requérir de façon continue devrait conduire les professionnels à établir plus clairement leurs objectifs d'intervention, les mécanismes de liaison avec d'autres établissements et à offrir, ce faisant, une meilleure garantie de la continuité de leurs interventions.

Les divers acteurs sociaux feront face à la nécessité de créer des passerelles entre services, professionnels, établissements et secteurs 
d'activité (collaboration avec les HLM ou le secteur résidentiel privé, mise sur pied de services alimentaires en concertation avec les organismes bénévoles ou publics, accès privilégié à l'hospitalisation ou à l'hébergement en cas d'urgence ou pour du répit, etc.). D'ores et déjà, de telles initiatives sont à l'œuvre lorsque les divers partenaires mettent leurs efforts au service du client et de leur communauté en facilitant I'orientation, en instaurant des mécanismes de partage d'information, d'expertise, de planification commune de leurs services.

\section{Assurer des services d'assistance, d'aide et de répit accessibles et de qualité}

L'article 478 de la Loi sur les services de santé et les services sociaux adoptée en 1991 autorise le ministre, une régie régionale, un établissement ou un organisme à fournir de l'aide matérielle ou financière pour le maintien à domicile d'une personne. Les conditions d'application seront prévues par règlement (article 480), notamment en ce qui concerne les situations de besoin, les revenus et les avoirs, les besoins considérés ou exclus dans la situation financière, les sommes qui peuvent être versées et les biens et services qui peuvent être fournis.

Reconnu légalement, le fait de subventionner un usager pour l'achat de services d'aide et d'assistance risque ainsi de s'étendre et, ce faisant, de nouvelles ressources dont les finalités sont très différentes pourront combler également le besoin de personnel pour assurer les services.

Il est probable que, dans les régions urbaines, les agences privées à but lucratif de services à domicile continueront de se développer. $\mathrm{D}^{\prime}$ autres organisations à but non lucratif, coopératives, regroupements de bénévoles, mais utilisant souvent des programmes de réinsertion sur le marché du travail, émergent également.

Cette fragmentation des services d'aide que l'on considère comme essentiels dans une stratégie de soutien à domicile appelle des choix pour le futur. Plusieurs scénarios sont envisageables:

- le retrait progressif du secteur public de ce champ au profit du secteur privé ou du secteur communautaire ;

- la transformation des pratiques internes des CLSC, notamment sur le plan des horaires de travail et de la souplesse des services, et un abaissement des coûts pour certaines tâches ;

- l'émergence de formules mixtes entre les services publics, communautaires ou privés;

- la tarification des services d'aide domestique selon le revenu des personnes. II n'est pas exclu de penser que dans la révision de 
la gamme des services assurés le gouvernement remette en question la couverture des services d'aide. C'est d'ailleurs le cas dans les nouvelles initiatives gouvernementales annoncées en 1994, soit les crédits d'impôt pour les aînés qui créeront des emplois familiaux et les Corporations intermédiaires de travail dans le secteur de l'aide à domicile qui s'autofinanceront partiellement par la contribution des clients.

Cet enjeu est central pour l'avenir du soutien à domicile. Si les tendances actuelles à l'éclatement des services se maintiennent, elles mettront en péril la valeur fondamentale d'un service public: le respect du droit des aînés vulnérables d'être soutenus par des personnes qualifiées et leur droit de recours advenant des insatisfactions. De plus, ils n'ont pas à porter seuls, s'ils ne le désirent pas, l'organisation parfois complexe des services.

$S^{\prime}$ il est essentiel d'élargir les options, celles-ci, à notre avis, doivent être mûrement réfléchies. Dans une optique où, de toute évidence, l'accès aux services publics sera plus sélectif, il est essentiel que la construction d'alliances avec la population, avec le secteur communautaire à but non lucratif et avec les élus locaux devienne une priorité à un double point de vue :

- l'élargissement, dans une stratégie concertée, de la couverture de services, tels l'entretien domestique, le déneigement, les réparations, l'entretien des extérieurs et le répit-gardiennage;

- I'intégration de ces services dans une stratégie de développement économique local par la création d'emplois de qualité dans la communauté et en CLSC tout en favorisant la réinsertion sociale.

\section{Maintenir des services publics de qualité et mieux adaptés}

Il existe une marge décisionnelle locale propice au réaménagement de l'organisation des services. Plusieurs solutions sont à la portée des intervenants et des gestionnaires des CLSC. Celles présentées ci-après s'inspirent d'une réflexion conduite à la Fédération des CLSC par un comité de travail en $1991^{15}$ et d'une étude récente effectuée par le $\mathrm{MSSS}^{16}$, portant sur l'organisation des services aux personnes âgées en perte d'autonomie par les CLSC et les centres hospitaliers.

15. FCLSCQ, Le soutien à domicile, document de réflexion $n^{\circ} 4$, avril 1991.

16. L. Trahan, L. Bélanger et M. Bolduc, Pour des services de qualité aux personnes âgées en perte d'autonomie. Une évaluation de la prestation de services dans les CLSC et les centres hospitaliers, MSSS, Direction de l'évaluation, juin 1993. 
a) Accorder la priorité aux services non spécialisés pour les besoins de base, dans une stratégie d'allocation des ressources

En effet, si les services de base pour les personnes qui ont des incapacités importantes n'ont pas un niveau minimal de qualité, de même que la souplesse, la régularité et l'intensité nécessaires pour répondre aux besoins des personnes, I'incitation au soutien à domicile sera réduite et le recours à l'hébergement public et privé ou, encore, le glissement vers des formules de services non supervisées s'accentueront.

Cette nécessité d'accessibilité conduit naturellement à envisager, au besoin, la mixité de services entre le secteur public et le secteur communautaire, par exemple sur le plan de l'aide domestique et du gardiennage, dans un cadre précis qui garantit le contrôle de la qualité des services, leur complémentarité et leur continuité.

b) Augmenter la flexibilité horaire

Cet aspect est une incontournable nécessité pour l'ensemble du personnel voué au maintien à domicile. Cependant, les services des auxiliaires familiales et les soins infirmiers sont particulièrement visés.

c) Améliorer la qualité du processus décisionnel dans l'évaluation, I'allocation et la détermination du plan de services. À cet égard, la personne concernée et sa famille doivent être clairement mises à contribution, et le plan d'intervention devra tenir compte, en premier lieu, de leurs besoins réels. Même si cette façon de procéder peut avoir pour conséquence la création de listes d'attente pondérées par le respect des critères de protection, elle nous semble plus respectueuse que la dilution de services. Enfin, le processus décisionnel interne doit se faire avec des outils normalisés d'évaluation et être appuyé par un fonctionnement multidisciplinaire. Si l'état des ressources ne permet pas une couverture appropriée des besoins, les critères de sélection doivent se fonder sur des paramètres objectifs communs entre CLSC et être connus de la population.

d) Miser sur la compétence des services publics de première ligne Il est essentiel de prendre conscience des principaux avantages pour la population d'avoir accès à des services à domicile publics: la finalité sociale des services, I'orientation des clientèles dans l'ensemble du système de santé et des autres ressources, la qualité d'un personnel bien formé, encadré et supervisé (et cela a un prix), la capacité multidisciplinaire d'intervention, la protection des 
personnes susceptibles de vivre des abus, le pouvoir d'engagement avec la communauté et l'imputabilité.

De plus, quand existent, au niveau local, dans l'entourage immédiat des personnes, des services informels d'entraide pouvant répondre aux attentes des personnes, des complémentarités réelles de rôles sont possibles. De ce fait, l'orientation et la liaison avec ces ressources devraient être considérées comme un rôle encore plus indispensable à assumer pour les CLSC.

\section{Faire du soutien à domicile un enjeu communautaire et politique}

Les services à domicile se retrouvent donc, de manière plus aiguë qu'auparavant, au confluent du système de santé et des solidarités familiales et sociales qui influenceront la qualité de vie des aînés. Dans ce cadre, il est clair que la mobilisation locale, régionale et provinciale autour de cet enjeu est désormais un des moyens essentiels pour sortir de l'impasse actuelle.

C'est d'ailleurs I'objectif qu'a poursuivi la coalition Vivre chez soi lors du forum qui réunissait en 1991 les associations représentant les aînés, les personnes handicapées, le secteur bénévole, le secteur municipal, celui de l'habitation, du transport et des CLSC. Une déclaration de principe a été entérinée par plus de 400 associations (regroupements d'aînés et de personnes handicapées, centres de bénévolat, offices municipaux, conseils municipaux, organismes locaux et CLSC) et présentée à tous les élus et au premier ministre en 1992.

Il s'agit ni plus ni moins de créer un large consensus sur le droit qu'a toute personne de rester dans son milieu, même si son autonomie est limitée, et d'être soutenue de façon digne et respectueuse.

Pour y parvenir, la seule possibilité est de construire, au cours des prochaines années, un réseau d'alliances autant sur le plan local, régional que provincial entre les usagers et les organismes qui les représentent, les élus et l'ensemble des fournisseurs de services qui œuvrent dans le champ du soutien à domicile dans une perspective de mieux-être de la population.

Cela nous paraît la condition sine qua non pour effectuer un virage majeur en faveur du soutien à domicile qui devrait comprendre:

- l'organisation locale des services en fonction des besoins des personnes; cette organisation comporte l'exploration de nouveaux modes de création de services non professionnels dans le cadre 
des mesures de lutte à la pauvreté et à la dépendance dans une stratégie concertée des divers ministères;

- la concertation de tous les organismes et des institutions publiques et privées concernées pour adapter et synchroniser leurs efforts en fonction des besoins diversifiés des aînés et de leurs proches, notamment de sécurité, de soutien ponctuel, d'un cadre résidentiel adapté, comme en témoigne le développement du secteur résidentiel privé associant logis et services;

- la sensibilisation à la valeur sociale et économique du soutien à domicile ;

- la mise en œuvre d'une politique interministérielle de soutien à domicile;

- un investissement financier majeur dans ce secteur par une réallocation significative des ressources institutionnelles et des mesures fiscales pertinentes.

\section{CONCLUSION}

Les services à domicile, malgré un intérêt croissant, n'ont jamais véritablement fait l'objet d'une réflexion particulière qui permette de les voir comme un élément essentiel du système socio-sanitaire ni, également, comme étant étroitement corrélés à un ensemble de mesures intersectorielles d'intégration sociale des personnes en perte d'autonomie. On doit le regretter, car dans les faits les aînés en pâtissent.

Lorsqu'on considère, dans les seuls termes financiers, c'est-à-dire par rapport aux budgets provinciaux ou par comparaison avec les autres provinces, la piètre considération dont ils font l'objet, il est difficile de ne pas voir là une profonde injustice et une distorsion institutionnelle majeure. Les stratégies d'adaptation sont déjà à l'œuvre, et elles peuvent entraîner des conséquences pénibles pour les usagers et dangereuses pour les établissements selon les modèles privilégiés.

Plusieurs solutions sont explorées. La première, celle d'un développement massif des services à domicile par le truchement de transferts intrasectoriels, notamment du secteur institutionnel vers la première ligne, mérite toute l'attention des décideurs locaux, régionaux et provinciaux. La seconde consiste en la transformation "culturelle» des modèles de pratiques professionnelles et de gestion pour respecter, soutenir et encourager le désir des personnes âgées et de leurs familles qu'elles restent chez elles, de même qu'en l'obligation pour les fournisseurs de services de mettre leurs ressources en synergie. Dans cette perspective, on ne devrait plus accepter au Québec qu'une personne 
soit hébergée ou hospitalisée faute de services adéquats dans son environnement familier. La troisième enfin, soit la nécessité de répondre à une demande plus forte de services d'aide et la volonté de ne pas abandonner les personnes âgées à des solutions de services non supervisées, impose le déploiement de services différenciés associant le secteur communautaire et le secteur public. La mise en œuvre de nouvelles stratégies associant la lutte à la pauvreté et la création d'emplois de qualité dans des services socialement utiles sur le plan local nous paraît une autre réponse prometteuse. À travers la mise en place de rôles complémentaires entre le CLSC, les organismes à vocation communautaire et les municipalités, il est possible de concilier le socialement souhaitable et l'économiquement utile.

Selon nous, ces trois options permettraient d'améliorer la qualité de vie des aînés dans la communauté et d'amorcer une action vigoureuse et immédiate en faveur du soutien à domicile. Si les CLSC saisissent ensemble les nouvelles possibilités et se donnent pour stratégie de renforcer les dynamiques communautaires, ils pourront aussi faire des citoyens leurs alliés dans la redistribution des ressources régionales et l'organisation des services sur le plan local.

Cette crise n'aura pas pour conséquence une crise des CLSC en tant qu'institution et sera l'occasion de retrouver l'intégration de toutes ses vocations initiales dans le meilleur intérêt de la population. 